in 11 babies (including a pair of twin). Caesarean operations were performed in 14 courses (including a pair of twin).

Conclusions In our hospital, we could well manage the course of pregnancy associated with SLE.

\section{FIRST REPORT OF INTRAUTERINE PERICARDIOCENTESIS IN A FETUS WITH NEONATAL LUPUS ERYTHEMATOSUS}

${ }^{1}$ MP Petersen* ${ }^{1}$ A Bygum, ${ }^{2} \mathrm{~A}$ Voss. ${ }^{1}$ Odense University Hospital, Dermatology and Allergy Centre, Odense, Denmark; ${ }^{2}$ Odense University Hospital, Rheumatology, Odense, Denmark

\subsection{6/lupus-2017-000215.394}

Background and aims Neonatal lupus erythematosus (NLE) is a passively acquired autoimmune disease of infancy, caused by the transplacental passage of maternal autoantibodies, mostly antiRO/SSA and anti-LA/SSB. NLE presents with a transient rash and/ or congenital heart block (CHB). The risk of developing NLE in SSA-positive women is $\sim 2 \%$, however the risk increases to $25 \%$, if the mother has had a previous child with NLE.

Objectives We present a case of NLE characterised by a thirddegree $\mathrm{CHB}$, ascites and life-threatening pericardial effusion, which was treated twice with intrauterine pericardiocentesis in week 22 and 29. After birth the child was treated with systemic corticosteroid on and off for 1 year, and she later received a pacemaker and was treated with ACE inhibitor and diuretics due to heart failure. Now, at the age of 6 years, she is still treated with ACE inhibitor. Her older sister also had NLE and her mother was found to have asymptomatic antiSSA $>100 \mathrm{U} / \mathrm{ml}$.

Discussion This case is exceptional, as the fetus had severe exudative pericarditis and had life-saving pericardiocentesis performed in utero. We want to draw the clinicians' attention to the increased risk of NLE, when a mother earlier has given birth to a child with NLE. Regular fetal echocardiography is important from week 16. In case of first- and second-degree $\mathrm{CHB}$, maternal corticosteroid can be tried to reverse the condition. Also, treatment with hydroxychloroquine or IVIG may decrease the risk of $\mathrm{CHB}$.

\section{MATERNAL AND FETAL OUTCOMES AMONG FILIPINO PATIENTS WITH SYSTEMIC LUPUS ERYTHEMATOSUS: A SINGLE CENTRE STUDY}

CA Dela Cruz-Tan*. University of Santo Tomas Hospital, Rheumatology, Manila, Philippines

\subsection{6/lupus-2017-000215.395}

Background and aims Systemic lupus erythematosus (SLE) usually affects women of child-bearing age, with pregnancy thus posing a relevant management challenge. This paper describes materno-fetal outcomes among Filipino SLE patients in a single tertiary care centre.

Methods We retrospectively reviewed the medical files of patients in the Lupus Database of the University of Santo Tomas Hospital in Manila, Philippines, who had a recorded pregnancy after SLE diagnosis, describing the maternal and fetal outcomes of each pregnancy.

Results There were a total of 197 pregnancies among 99 patients. Average age at first pregnancy was 29 years old. Lupus Nephritis (LN) was present in 13.7\%, hyperthyroidism $1.0 \%$, and autoimmune thyroiditis $1.0 \%$. Maternal complications intra- and post-partum included hypertension (5.6\%), pulmonary tuberculosis (TB) $(4.0 \%)$, pre-eclampsia $(3.6 \%)$,
HELLP syndrome, gestational diabetes (GDM) (3.0\%), urinary tract infections (UTI) (3.0\%), herpes zoster (2.0\%) and $0.5 \%$ cases each of TB meningitis, TB spondylitis, dilated cardiomyopathy, and postpartum depression. Term deliveries were recorded in 98 (79.0\%) pregnancies while 26 (21.0\%) were delivered preterm. There were $73(37.0 \%)$ nonviable pregnancies including miscarriages (82.2\%), intrauterine fetal demise (IUFD) (11.0\%) and blighted ovum (6.8\%). Normal birth weight was recorded in $83.9 \%$ of infants. Congenital abnormalities included congenital heart block $(0.8 \%)$, meningocoele $(0.8 \%)$, thyroid abnormality $(0.8 \%)$, G6PD deficiency $(0.8 \%)$, and autism (0.8\%).

Conclusions Although successful pregnancy outcomes are possible for SLE patients, miscarriages, preterm deliveries, blighted ovum and IUFD remain a concern, requiring close monitoring and intensive multi-specialty team approach.

\section{SLE Epidemiology and risk factors}

\section{QUALITY OF LIFE OF PATIENTS WITH LUPIC NEPHRITIS: THE CASE OF THE COLOMBIAN CARIBBEAN REGION}

G Aroca Martínez*, HJ Gonzalez-Torres, A Martinez. Universidad Simon Bolivar, Atlántico, Barranquilla, Colombia

\subsection{6/lupus-2017-000215.396}

Background and aims The objective of this study was to analyse the impact of lupus nephritis (NL) on the quality of life of 30 patients from the coast clinic in the city of Barranquilla, Colombia, diagnosed with this disease.

Methods To evaluate the patients' quality of life, the GENCAT scale was used as the instrument of study and three controls were performed on each patient since they were diagnosed with NL. It also counted with the collaboration of the medical and psychological body of the clinic.

Results The results of the study showed that the patients in the first controls obtained a low score with respect to the dimensions that make up the GENCAT scale, which revealed that the patients did not perceive a satisfactory quality of life. From the second and third controls, it was possible to observe improvements in the dimensions of the GENCAT scale, indicating that the patients began to perceive a favourable quality of life.

Conclusions When the patient has a good response to treatment, aspects of their quality of life improve markedly

\section{CLINICAL-PATHOLOGICAL CHARACTERISATION OF COHORT OF 400 PATIENTS WITH LUPUS NEPHRITIS: NEFRORED REGISTRY}

${ }^{1,2} \mathrm{G}$ Aroca Martínez, ${ }^{1,2} \mathrm{~A}$ Cadena Bonfanti, ${ }^{1} \mathrm{~A}$ Domínguez Vargas, ${ }^{1} \mathrm{D}$ Silva Díaz, ${ }^{1} \mathrm{~A}$ Acuña Feyte, ${ }^{1} \mathrm{H}$ González Torres*, ${ }^{3} \mathrm{~A}$ Iglesias Gamara, ${ }^{4} \mathrm{E}$ Egea Bermejo. ${ }^{1}$ Universidad Simon Bolivar, Medicine, Barranquilla, Colombia; ${ }^{2}$ Clínica de la Costa, Nephrology, Barranquilla, Colombia; ${ }^{3}$ Universidad Nacional de Colombia, Medicine, Bogotá DC, Colombia; ${ }^{4}$ Universidad del Norte, Medicine, Barranquilla, Colombia

\subsection{6/lupus-2017-000215.397}

Background and aims Lupus Nephritis is one of the most serious complications of SLE with a prevalence of $65 \%$, it is diagnosed in the first five years of SLE and is uncommon after 10 years. Between $5 \%$ and $25 \%$ of cases have Chronic Renal Disease (CKD) after 5 years. 
Methods NEFRORED begins in 2008 as a multicenter registry of prospective observational cohorts, which includes patients with confirmed diagnosis of NL through renal biopsy. Study the incidence of the clinical and pathological characteristics of NL in the Colombian Caribbean region. The database was implemented with the variables of the clinical records of the patients.

Results Eight centres were involved and 400 patients have been included, $88 \%$ female; ratio between men and women is 7:1 with a median age 37 years. Urinary parameters have evidenced 24 hours mean proteinuria of $1.9 \pm 3.3 \mathrm{~g} / 24$ hours and microscopic hematuria in 50\% of patients. Among nephrological syndromes, nephritic syndrome and hematuria-proteinuria have been the most frequent $70 \%$, followed by nephrotic syndrome and non-nephrotic proteinuria 30\%. Hypocomplementemia is evidenced in $60 \%$ of the patients. The median of the SLEDAI has been 10.2 points. Antinuclear Antibodies are positive in $90 \%$. NL class IV-G (A) has been the most frequent $75 \%$. Chronic Renal Disease is concomitant in $52 \%$ of the cases.

Conclusions NEFRORED represents the first Colombian cohort of patients diagnosed with NL, with up-to-date information on the renal manifestations of SLE, comorbidities, disease status and clinical evolution under conditions of real clinical practice. CKD remains a significant comorbidity in patients with NL.

\section{USE OF TICS FOR THE GENERATION OF A MODEL OF MANAGEMENT AND FOLLOW-UP OF PATIENTS WITH LUPIC NEPHRITIS}

${ }^{1,2} \mathrm{G}$ Aroca Martínez, ${ }^{1,2} \mathrm{~A}$ Cadena Bonfanti, ${ }^{1} \mathrm{H}$ Gonzalez Torres* ${ }^{3} \mathrm{~K}$ Moreno, ${ }^{3} \mathrm{M}$ Garay Palacio, ${ }^{3} \mathrm{G}$ Palacio Betancourt. 'Universidad Simon Bolivar, Medicine, Barranquilla, Colombia; ${ }^{2}$ Clinica de la Costa, Nephrology, Barranquilla, Colombia; ${ }^{3}$ TIC Social, Project, Bogota D.C., Colombia

\subsection{6/lupus-2017-000215.398}

Background and aims Our objective was to create a model for the characterisation, classification and management, according to the protocols, of patients with lupus nephritis, which is done in real time and with the generation of alerts at the right time, which allows inferring that we will obtain a Better quality of life of the patients, reduction of complications and, therefore, decrease in the cost of handling these patients.

Methods Initially patients with NL were classified with the CCM system for the risk of the disease. With the help of the CCM information tools, protocols of care and follow-up were incorporated into the software, which made it possible to manage, monitor and control patients.

Results The management of NL patients was handled by a group of specialists whose ultimate goal was to improve the quality of life and identify possible deterioration in the patient's health. In an integral way with CCM, a work plan was determined for each of them, keeping a strict record of the results of examinations that allowed to take medical actions in favour of the patients. Also, a mobile application (CheckUp!) is made available to the user in order to monitor the patient in real time with NL.
Conclusions It was established a flow of actions to manage alterations in the quality of life of the patient. Actions that led to the change in the risk category of the disease per patient and a greater adherence and incorporation to the management to the family nucleus, making the model a success.

\section{THE RELATIONSHIP BETWEEN SOCIOECONOMIC FACTORS AND IMMUNOSUPPRESSIVE USE IN SYSTEMIC LUPUS ERYTHEMATOSUS}

${ }^{1} \mathrm{~A}$ Bankole*, ${ }^{2} \mathrm{~S}$ Ford, ${ }^{2} \mathrm{~F}$ Wong, ${ }^{2} \mathrm{~J}$ McMunn, ${ }^{2} \mathrm{SS}$ Zaman, ${ }^{2} \mathrm{~J}$ Rawlins. ${ }^{1}$ Carilion Clinic, Rheumatology, Roanoke, USA; ${ }^{2}$ Carilion Clinic, Internal medicine, Roanoke, USA

\subsection{6/lupus-2017-000215.399}

Background and aims Systemic Lupus Erythematosus (SLE) is more common in minorities, who also tend to be more socially disadvantaged. Some studies have shown a disparity in access to care and medication prescribed in minorities and socially disadvantaged people with SLE. The purpose of this study was to examine the association between socioeconomic factors including gender, race, and median household income and medication prescribed in our SLE cohort.

Methods The study design was a retrospective cohort single centre study. A review of electronic medical record yielded 115 patients that were included in the cohort. General demographic information including age, gender, zip code and serological testing was obtained through chart review and recorded in the database. Supplemental socioeconomic information for each zip code in Southwest Virginia was obtained from the United States Government Census website. The data was analysed by Kruskal-Wallis Test, Mann-Whitney U Test using SAS9.3.

Results Among the 113 patients, there was no relationship between socioeconomic factors and medication prescribed. There was no statistical relationship between gender and medication prescribed. There was no statistical relationship between race and medications. More importantly, no statistical relationship exists between median household income and medications prescribed.

Conclusions There was no relationship between socioeconomic factors and the medications prescribed in SLE within our cohort. This may relate to treatment standardisation of SLE and the increasing awareness of the "treat to target" ethos in rheumatology. The limitations of this study include the small sample size and it's restriction to a single centre.

\begin{tabular}{|c|c|c|}
\hline \multicolumn{2}{|c|}{ Sex and Racial Distribution } \\
\hline & $N$ & Percent \\
\hline Female & 95 & $87.2 \%$ \\
\hline male & 14 & $12.8 \%$ \\
\hline Caucasian Americans & 73 & $67.0 \%$ \\
\hline African Americans & 28 & $25.7 \%$ \\
\hline Hispanic Amercains & 2 & $1.8 \%$ \\
\hline Other (not included in & 6 & $5.5 \%$ \\
\hline
\end{tabular}

